\title{
Pancreatic Carcinoma Associated with Chronic Pancreatitis
}

\author{
Hideyuki WaKasugi, Akihiro Funakoshi, Haruo Iguchi, Motoharu Takase, Masanobu Inoue, \\ Akira OHSHIMA and Yousuke SeO
}

\begin{abstract}
The incidence of invasive ductal carcinoma of the pancreas was $3.1 \%$ (6 cases) in 196 patients with definite chronic pancreatitis. Five patients ( 3 men and 2 women) had calcific pancreatitis and 1 patient (man) had non-calcific pancreatitis. Large pancreatic stones were recognized in 2 women. Most of the patients complained of continuous intractable abdominal pain and/or back pain together with weight loss and appetite loss. Serum CA19-9 levels and exacerbation of glucose intolerance were retrospectively noted to have been elevated in 1 patient. However, it was difficult to obtain a definitive diagnosis by imaging examinations earlier, due to the presence of chronic pancreatitis. Median survival of the 6 patients was 6.5 months from admission. (Internal Medicine 38: 951-956, 1999)
\end{abstract}

Key words: coincidence, pancreatic stones, follow-up, obstructive pancreatitis, definitive diagnosis, survival

\section{Introduction}

Patients with chronic pancreatitis are reported to have a higher prevalence of pancreatic carcinoma than the general population $(1,2)$. Recognition of carcinoma in patients with chronic pancreatitis is considered difficult (3). To evaluate the relationship between chronic pancreatitis and pancreatic carcinoma, we examined patients with chronic pancreatitis and reviewed patients in whom pancreatic carcinoma was found.

\section{Case Reports}

We investigated 6 cases of chronic pancreatitis associated with adenocarcinoma of the pancreas of duct-cell origin (invasive ductal carcinoma) that were treated at our hospital to clarify the clinical features of such patients (Tables 1-4). Chronic pancreatitis was diagnosed according to the 1983 criteria of the Japan Pancreas Society (4). The criteria were revised in 1995 (5), but the diagnosis in these patients was not affected by the change in criteria. In brief, one or more of the following criteria were fulfilled: histological findings compatible with chronic pancreatitis; detection of pancreatic calcification; abnormal exocrine pancreatic function on the pancreozymin/ secretin test or the secretin test; and abnormalities of the pancreatic duct and/or pancreas on imaging studies. Clinical diagnosis of pancreatic carcinoma was made based on imaging examinations and the clinical course. Endocrine pancreatic function was evaluated by oral glucose tolerance test.

Case 1: A 48-year-old man underwent surgery for suspected pancreatic cancer of the body and tail 32 months previously (distal pancreatectomy). At that time, carcinoma was not found but pancreatolithiasis was detected. No follow-up after that. Then, our examination revealed an abdominal tumor due to pancreatic carcinoma as well as a liver tumor which was diagnosed as adenocarcinoma. The patient died due to cachexia and dyspnea (lung metastasis) 5 months later.

Case 2: A 49-year-old woman, who complained of back pain, was admitted for further examination of pancreatolithiasis (large stones) in the head of the pancreas. In addition to imaging, biopsy of the duodenum showed carcinoma of the head of the pancreas with invasion of the duodenum, for which pancreaticoduodenectomy (PD) and radiation therapy (EBRT, external beam radiation therapy) were performed. The patient died of gastrointestinal bleeding 16 months postoperatively.

Case 3: A 63-year-old man with chronic alcoholic pancreatitis complained of appetite loss and upper abdominal pain (6). At that time, CT examination showed a cystic mass at the splenic hilum. He died 5 months later due to peritonitis carcinomatosis (adenocarcinoma of the tail of the pancreas).

Case 4: A 42-year-old woman complained of upper abdominal discomfort. Abdominal CT showed large stones in the pancreatic head and a tumor-like lesion in the pancreatic body. The latter disappeared 5 weeks later. However, nausea and weight loss developed. Balloon ERCP (endoscopic retrograde

From the Department of Gastroenterology, National Kyushu Cancer Center, Fukuoka Received for publication February 22, 1999; Accepted for publication July 14, 1999

Reprint requests should be addressed to Dr. Hideyuki Wakasugi, the Department of Gastroenterology, National Kyushu Cancer Center, 3-1-1 Notame, Minamiku, Fukuoka 811-1395 
WAKASUGI et al

Table 1. Clinical Findings in Pancreatic Carcinoma Patients Associated with Chronic Pancreatitis I

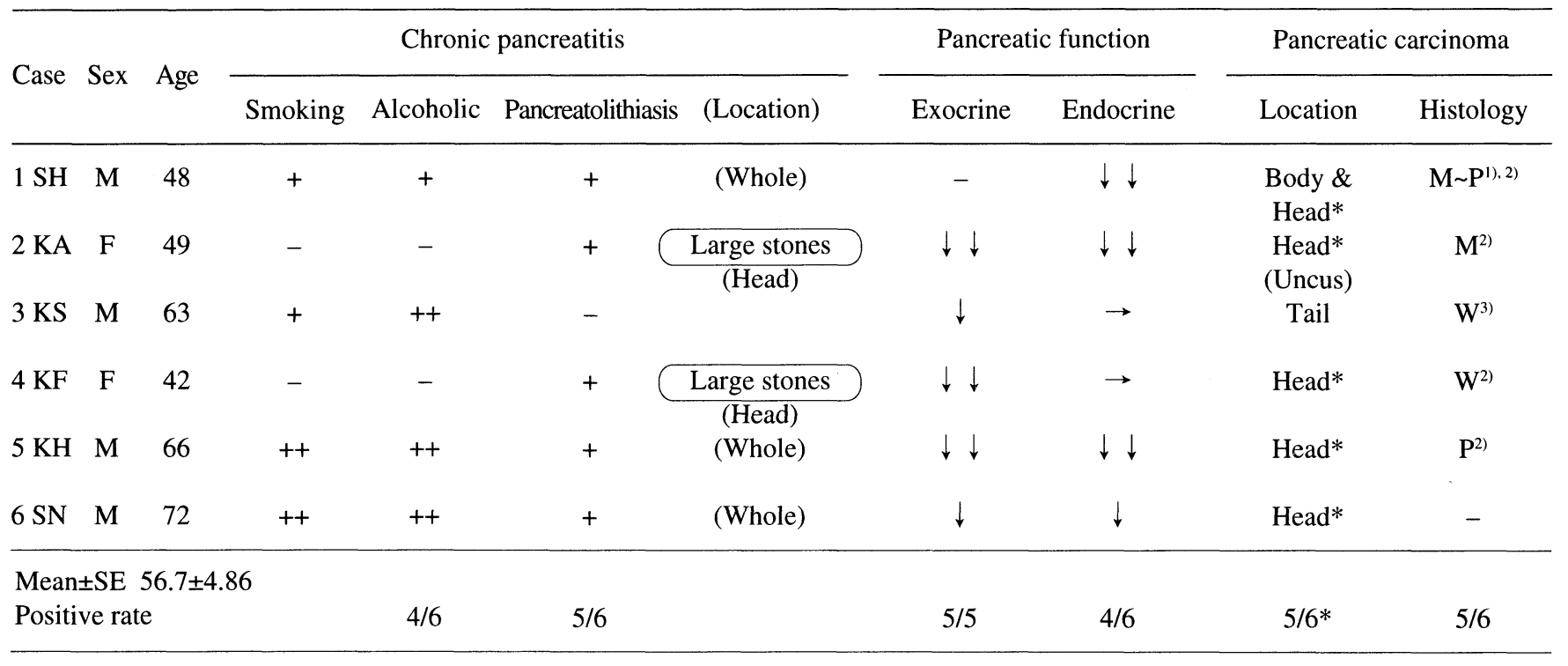

+Presence, -Absence, $\downarrow$ Decreased, $\rightarrow$ Within normal limits. * Ratio of pancreas head carcinoma patients to all carcinoma patients. ${ }^{1)}$ Biopsy (Liver), ${ }^{2)}$ Operation, ${ }^{3)}$ Autopsy. W: Well, M: Moderately, P: Poorly differentiated adenocarcinoma (All were invasive ductal carcinomas). Characteristic feature.

Table 2. Clinical Findings in Pancreatic Carcinoma Patients Associated with Chronic Pancreatitis II

\begin{tabular}{|c|c|c|c|c|c|c|c|}
\hline \multirow{2}{*}{ Case } & \multicolumn{6}{|c|}{ Around admission time } & \multirow{2}{*}{$\begin{array}{c}\text { After admission } \\
\begin{array}{c}\text { Abnormal } \\
\text { imaging }\end{array}\end{array}$} \\
\hline & $\begin{array}{c}\text { Abdominal } \\
\text { and/or } \\
\text { back pain }\end{array}$ & $\begin{array}{c}\text { Weight } \\
\text { (Appetite) } \\
\text { loss }\end{array}$ & $\begin{array}{l}\text { Physical } \\
\text { findings }\end{array}$ & $\begin{array}{c}\text { Serum } \\
\text { pancreatic } \\
\text { enzyme }\end{array}$ & $\begin{array}{l}\text { Blood } \\
\text { glucose } \\
\text { control }\end{array}$ & $\begin{array}{l}\text { Serum } \\
\text { tumor } \\
\text { marker }\end{array}$ & \\
\hline $1 \mathrm{SH}^{*}$ & + & + & Tumor & $\begin{array}{l}\text { Elevated } \\
\text { amylase }\end{array}$ & Poor & $\begin{array}{c}\text { Elevated } \\
\text { CEA }\end{array}$ & $\mathrm{US}, \mathrm{CT}$ \\
\hline $2 \mathrm{KA}$ & + & + & - & - & Poor & - & $\frac{\text { US, CT }}{\text { ERP (Biopsy) }}$ \\
\hline $3 \mathrm{KS}$ & + & - & Tumor & - & - & $\begin{array}{l}\text { Elevated } \\
\text { CA19-9 } \\
\end{array}$ & CT (Cystic mass) \\
\hline $4 \mathrm{KF}$ & \pm & + & - & - & - & - & $\frac{\text { Balloon ERP }}{\text { Duodenography }}$ \\
\hline $5 \mathrm{KH}$ & ++ & + & $\begin{array}{c}\text { Bruit } \\
\text { Knock pain }\end{array}$ & - & Poor & - & $\begin{array}{c}\text { US,CT } \\
\text { ERP, Angiography }\end{array}$ \\
\hline $6 \mathrm{SN}$ & ++ & + & Knock pain & $\begin{array}{l}\text { Decreased } \\
\text { elastase } 1\end{array}$ & Poor & $\begin{array}{l}\text { Elevated } \\
\text { CA19-9 }\end{array}$ & $\begin{array}{l}\text { CT } \\
\text { ERP }\end{array}$ \\
\hline $\begin{array}{l}\text { Rate of } \\
\text { abnormality }\end{array}$ & $6 / 6$ & $5 / 6$ & $4 / 6$ & $2 / 6$ & $4 / 6$ & $3 / 6$ & $6 / 6$ \\
\hline
\end{tabular}

US: Ultrasonography, CEA: Carcinoembryonic antigen, CT: Computed tomography, CA19-9: Carbohydrate antigen 19-9, ERP: Endoscopic retrograde pancreatography. *Second admission time. + Presence, - Absence. $\square$ Useful for diagnosis of the patient.

pancreatography) (7), which is expected to clarify the small pancreatic ducts, showed findings compatible with carcinoma of the pancreatic head which was considered nonresectable. Although radio-chemotherapy was performed, the patient died of cachexia 7 months after laparotomy.

Case 5: A 66-year-old man with chronic alcoholic calcific pancreatitis and diabetes, complained of severe continuous abdominal pain. He had not drunk much alcohol for more than 15 years. Diabetes had been treated by insulin. Bruit was recognized in the upper abdomen. Imaging showed a solid mass in the head of the pancreas and an encasement of the proper hepatic artery, which was stenotic but not long, suggesting a 
Table 3. Clinical Course in Pancreatic Carcinoma Patients Associated with Chronic Pancreatitis I

\begin{tabular}{|c|c|c|c|c|c|}
\hline $\begin{array}{l}\text { (1) Survival Period } \\
\text { (2) Date }\end{array}$ & $\begin{array}{l}\text { (1) (5) } \\
\text { (1) }\end{array}$ & $\begin{array}{l}\text { (2) (5) } \\
\text { (2) }\end{array}$ & $\begin{array}{l}\text { (3) } \sim(5) \\
\text { (3) }\end{array}$ & $\begin{array}{l}\text { (4) (5) } \\
\text { (4) }\end{array}$ & (5) \\
\hline \multirow[t]{2}{*}{$1 \mathrm{SH}$} & 36 & 2.5 & 2.5 & 1 & \\
\hline & '79-5- & ' $82-3-9 *$ & ' $82-3-13$ & ' $82-4-22$ & ' $82-5-22$ \\
\hline \multirow[t]{2}{*}{$2 \mathrm{KA}$} & 19.5 & 17.5 & 17 & 16 & \\
\hline & '83-7- & '83-9-1 & '83-9-13 & '83-10-17 & $85-2-15$ \\
\hline \multirow[t]{2}{*}{$3 \mathrm{KS}$} & 160 & 4.5 & 3.0 & 2.5 & \\
\hline & '74-3- & ' $87-2-23$ & '87-4-9 & '87-4-24 & '87-7-12 \\
\hline \multirow[t]{2}{*}{$4 \mathrm{KF}$} & 13 & 12 & 7.5 & 7.5 & \\
\hline & '91-4-20 & '91-5-27 & '91-10-8 & '91-10-8 & '92-5-27 \\
\hline \multirow[t]{2}{*}{$5 \mathrm{KH}$} & 260 & 5 & 5 & 4 & \\
\hline & '73-10-25 & '95-1-17 & '95-1-25 & '95-2-16 & '95-6-18 \\
\hline \multirow[t]{2}{*}{$6 \mathrm{SN}$} & 71.5 & 8 & 6 & 4.5 & \\
\hline & '91-12-4 & '97-3-21 & '97-5-19 & '97-6-3 & '97-11-23 \\
\hline $\begin{array}{l}\text { Median } \\
\text { survival }\end{array}$ & 53.8 & 6.5 & 5.5 & 4.25 & \\
\hline
\end{tabular}

(1) months, (2) year-month-day. (1) Diagnosis of chronic pancreatitis, (2) Admission, (3) Definitive diagnosis of pancreatic carcinoma, (4) Start of treatment for carcinoma, (5) Death. *Second admission. Characteristic feature.

Table 4. Clinical Course in Pancreatic Carcinoma Patients Associated with Chronic Pancreatitis II

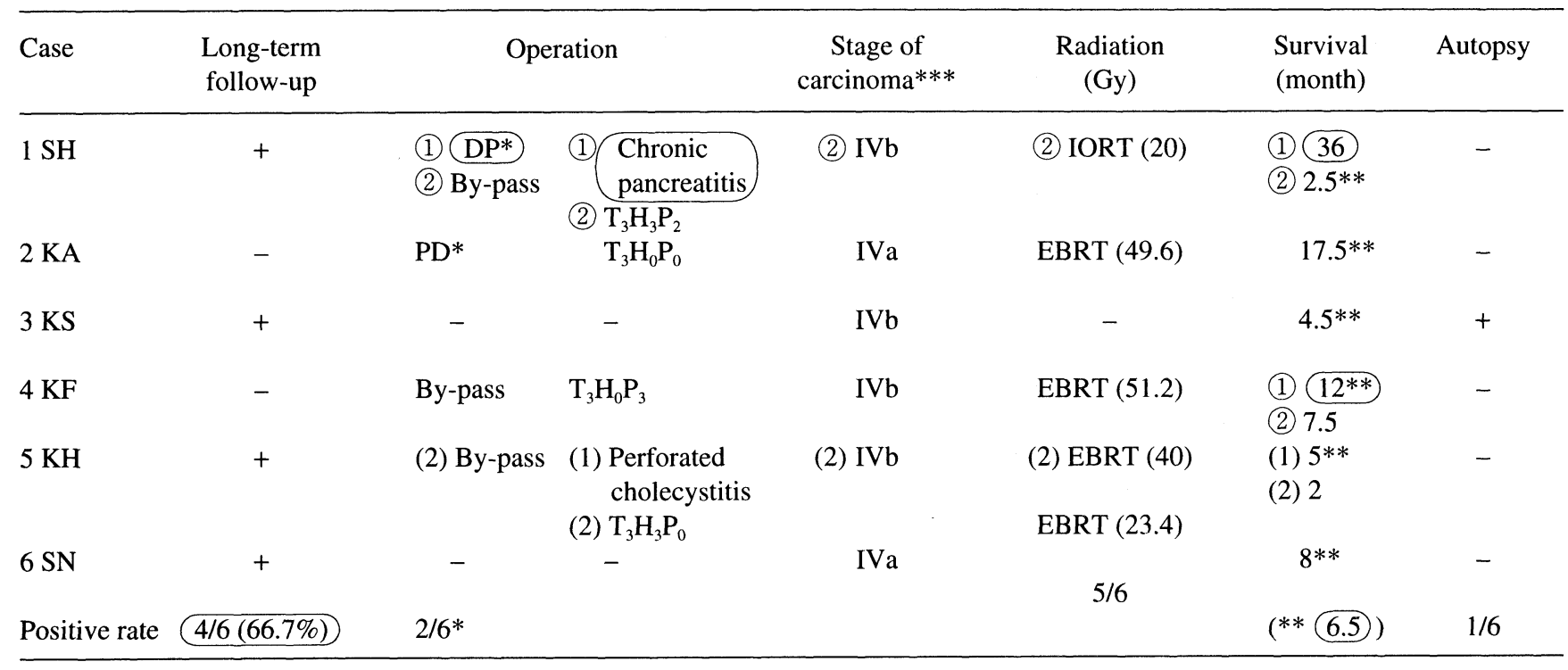

PD: Pancreaticoduodenectomy, IORT: Intraoperative radiation therapy, DP: Distal pancreatectomy, EBRT: External beam radiation therapy. (1) First admission (Carcinoma was suspected but chronic pancreatitis alone was recognized in case 1). (2) Second admission. (1) First operation, (2) Second operation. + Presence, - Absence. ***Japan Pancreas Society classification, 1993. C Characteristic feature.

nonresectable tumor (Fig. 1). Considerable calcification was observed within and around the mass lesion (Fig. 1). The patient died 8 months after initial consultation despite radio-chemotherapy.

Case 6: A 72-year-old man with chronic alcoholic calcific pancreatitis and diabetes, complained of increased abdominal and back pain. The diagnosis of cancer in the pancreatic head was initially difficult, but CT reexamination showed an invasive growth of the tumor in the body of the pancreas with lymph node swelling, which was not detected by CT examination 2 months before. Serum CA19-9 levels were noted retrospectively to be somewhat elevated compared to previous data (Fig. 2). Glucose intolerance progressed by the time a definitive diagnosis of pancreatic cancer was made (Fig. 3). Chemo-radiation was effective, but the patient died 8 months later.

The incidence of pancreatic carcinoma associated with chronic pancreatitis was $3.1 \%$ in 196 definite chronic pancreatitis patients ( 157 men and 39 women) examined over a $26-$ 
year period (1972-1997). Past history of chronic pancreatitis was found in 6 patients $(1.7 \%)$ out of 353 pancreatic carcinoma patients [ 224 men and 129 women, $62.1 \pm 0.62$ (mean \pm SE) years old] during the same period at our hospital. The mean

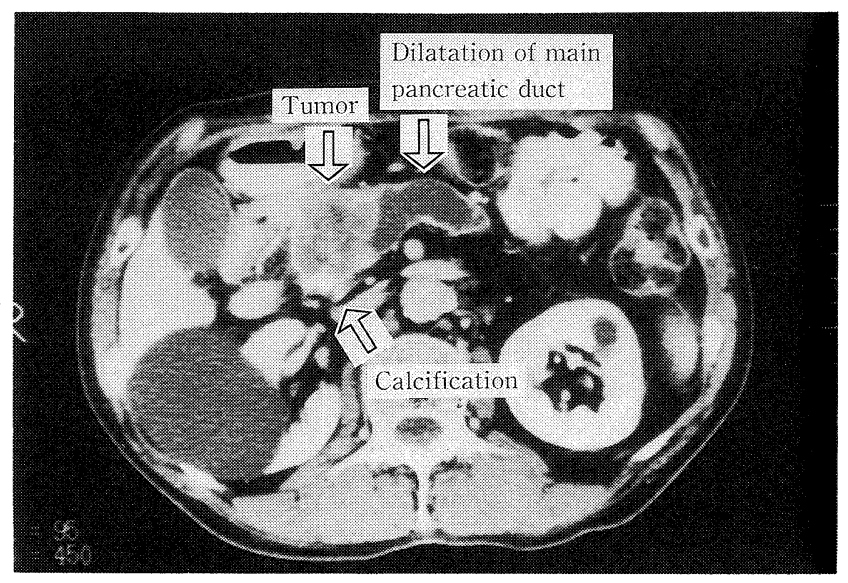

Figure 1. Abdominal computed tomography (CT) showing a high-density area in the head of the pancreas (arrow) enhanced with contrast medium, together with calcification around the area (arrow) and with dilatation of the main pancreatic duct in the distal part of the pancreas (arrow) in case KH. age of these 6 patients was 56.7 years. Five patients ( 3 men and 2 women) had calcific pancreatitis and 1 patient (man) had non-calcific pancreatitis (Table 1). Pancreatic diabetes was recognized in 4 patients with calcific pancreatitis (Table 1). Large pancreatic stones in the head of the pancreas were recognized in 2 women ( 49 and 42 years of age) (Table 1). These 2 patients did not habitually drink or smoke (Table 1). Most of the patients complained of continuous intractable abdominal and/ or back pain or abdominal discomfort, somewhat different from the complaints of chronic pancreatitis, together with weight loss and appetite loss (Table 2). Pancreatic tumor was suggested by imaging examinations performed in response to these complaints (Table 2). However, it was difficult to obtain a definitive diagnosis earlier, due to the presence of chronic pancreatitis, especially pancreatic stones (Table 3 ). Considerable calcification was shown within and around the mass lesion in the pancreas of 1 patient (Fig. 1), while a cystic mass was detected in 1 other patient. Serum CA19-9 levels were retrospectively noted to have been elevated compared with previous data in 1 patient (Fig. 2). Figure 3 shows that pancreatic diabetes may have exacerbated by September 1996. Four of the 6 patients were followed for a long period as chronic pancreatitis patients, and the stage of pancreatic carcinoma was advanced (Stage IV) when malignancy was identified (Tables 3 \& 4). Radiation therapy was performed in 5 out of 6 patients (Table 4). As a result, the median survival of the 6 patients was 6.5 months from admission (Tables $3 \& 4$ ).

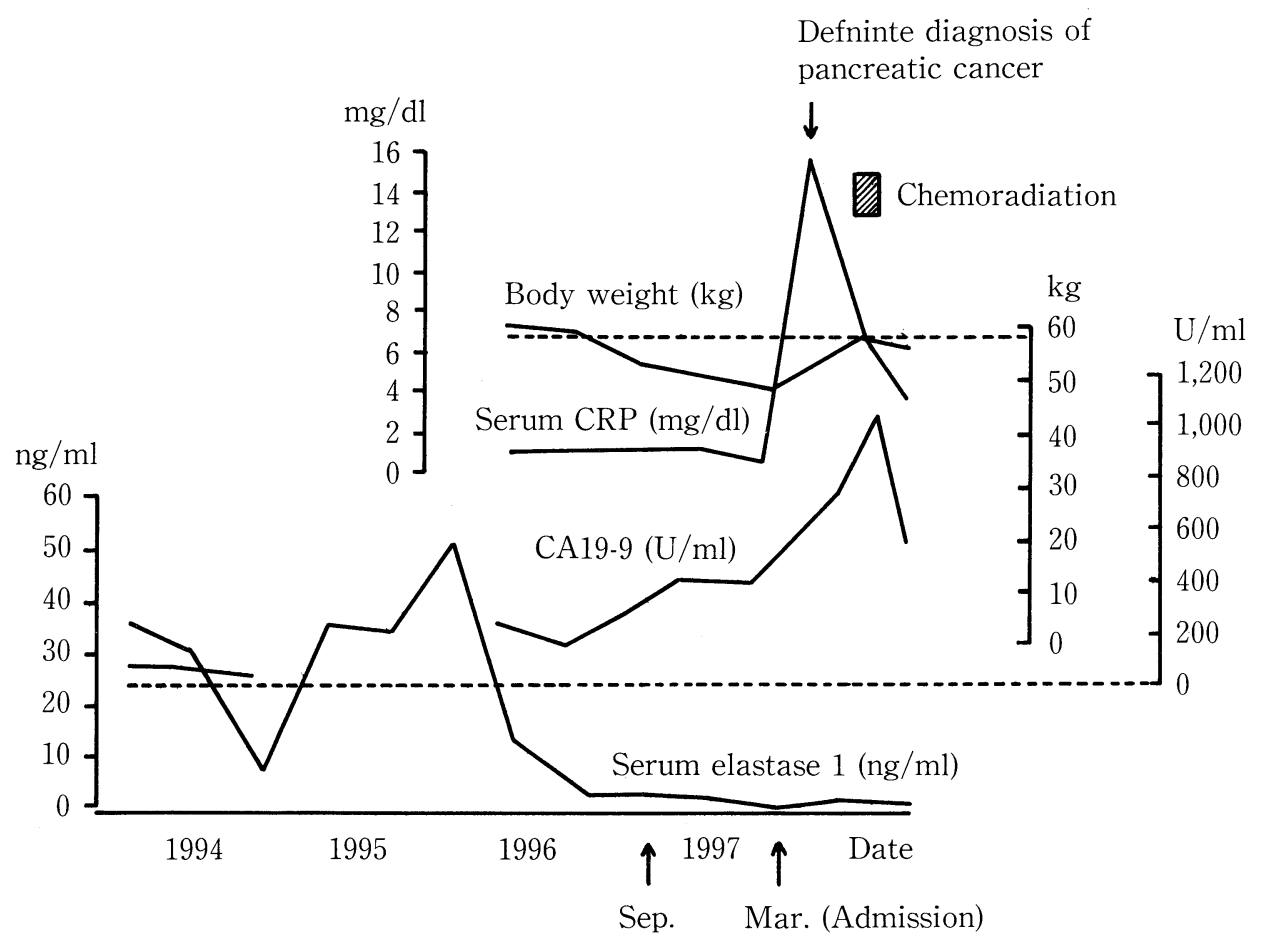

Figure 2. Follow-up data on laboratory findings in a patient with pancreatic cancer (SN). Serum CA19-9 levels continued to increase until the period of chemoradiation, while serum elastase 1 had decreased. The data suggest that obstructive pancreatitis had occurred around September 1996. 


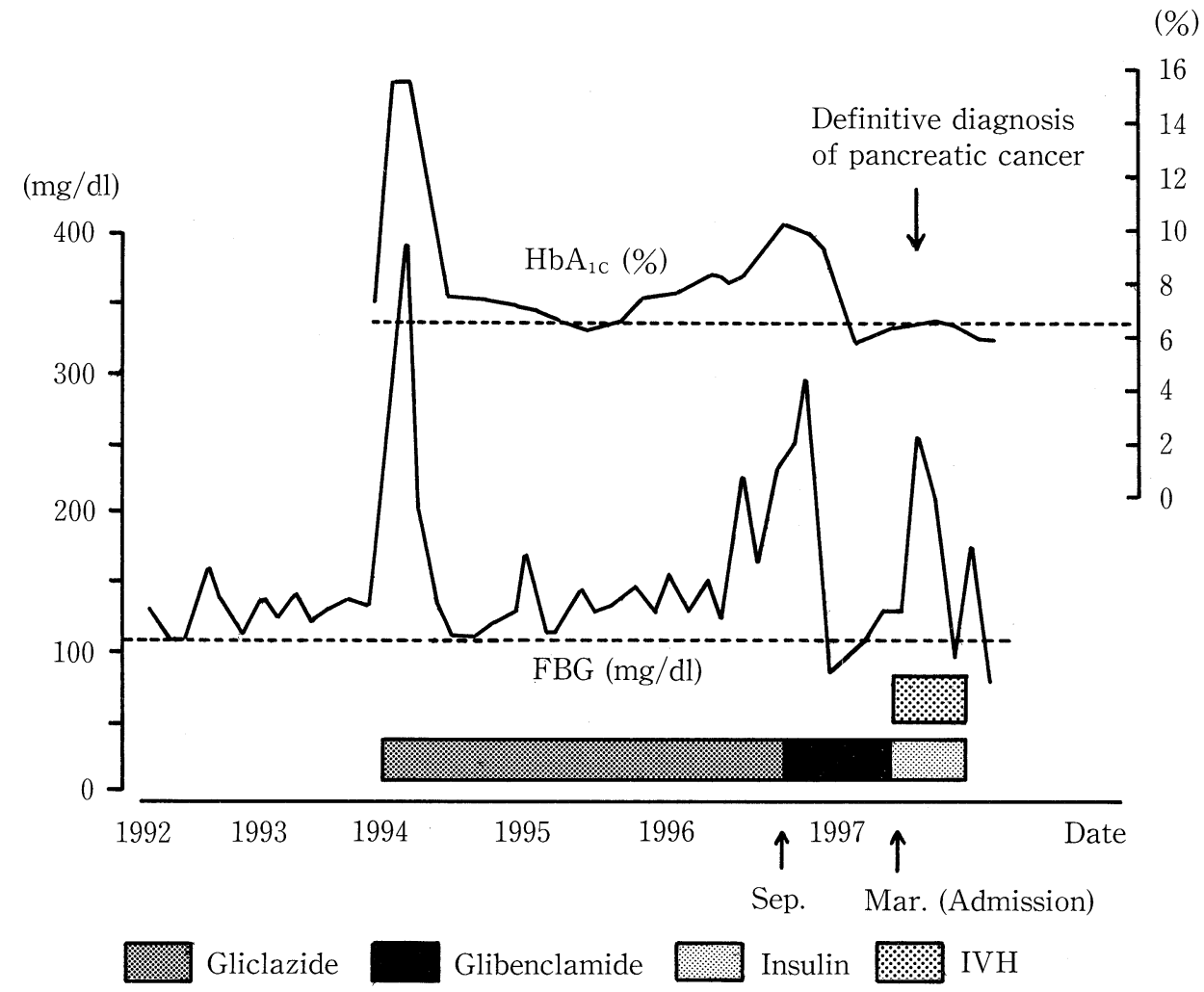

$\mathrm{HbA}_{1 \mathrm{c}}$ : Hemoglobin $\mathrm{A}_{1 \mathrm{c}}$, FBG: Fasting blood glucose, IVH: Intravenous

hyperalimentation

Figure 3. Blood glucose levels and treatment for diabetes in a pancreatic cancer patient (SN). The poor control of diabetes suggests obstructive pancreatitis caused by pancreatic carcinoma around September 1996.

Among the other 190 patients, there were 104 calcific and 86 non-calcific chronic pancreatitis patients. We could not generally find in these patients the above-mentioned severe continuous abdominal and/or back pain, weight loss and appetite loss, suddenly decreased serum elastase 1 and increased blood glucose values, and steadily increasing serum CA19-9 values.

\section{Discussion}

Lowenfels et al (1) reported that the cumulative incidence of pancreatic cancer was 1.8 percent and 4.0 percent, 10 and 20 years after the diagnosis of pancreatitis in 1,552 chronic pancreatitis patients, respectively. Yamaguchi (2) reported 4 pancreatic carcinoma patients associated with chronic calcifying pancreatitis with an incidence of $2.5 \%$ in 160 patients with pancreatic carcinoma. All 4 patients died within 14 months after the clinical diagnosis of pancreatic carcinoma. Our study showed that the incidence of pancreatic carcinoma associated with chronic pancreatitis was $3.1 \%$ in 196 patients with chronic pancreatitis. If the mean follow-up period is about 13 years, this ratio is compatible with the above-mentioned study (1). The coincidence of the two diseases was found in $1.7 \%$ out of pancreatic carcinoma patients at our hospital.

Thus, chronic pancreatitis has been proposed as a causal risk factor for pancreatic cancer. However, the relationship between chronic pancreatitis and pancreatic cancer remains controversial (8). A K-ras gene mutation in patients with chronic pancreatitis is not likely related to the development of pancreatic cancer during the follow-up period (9). Suehara et al (10) revealed that telomerase activity in pancreatic juice differentiates adenocarcinoma from adenoma and pancreatitis and may serve as a useful diagnostic marker. The present study showed that it is important to note the existence of large pancreatic stone, severe continuous abdominal and/or back pain, weight loss probably due to appetite loss, change of laboratory findings such as serum elastase 1, glucose and CA19-9 levels. A comparatively sudden decrease of serum elastase 1 levels suggested obstructive pancreatitis caused by pancreatic carcinoma, and exacerbation of glucose intolerance may have occurred due to the obstructive pancreatitis. However, establishing an early definitive clinical diagnosis is very difficult and the prognosis is poor in such patients, as previously reported (2). Current methods of diagnosing pancreatic cancer are difficult and of little use in patients with chronic pancreatitis (11). 


\section{WAKASUGI et al}

Definitive diagnosis for treatment must be made together with imaging examinations, these types of diagnostic methods remain unsatisfactory despite recent progress. Evaluation of the differential diagnosis between chronic pancreatitis and pancreatic carcinoma using imaging techniques such as US, CT, ERP, and angiography, revealed the following points to be important: careful follow-up observation of mass lesions, appearance of lymph node swelling, precise visualization of pancreatic ducts and blood vessels, and combined examination with biopsy.

On the other hand, Machiki et al (12) reported that pancreatic cancer associated with pancreatic stones was found in 4 (3.0\%) of 134 cases of pancreatic cancer resected at their hospital, and that 3 of these cases exhibited pancreatic cancer prior to pancreatic stones. Two of our patients, who had not been followed for a long period, showed a pattern of large pancreatic stones as reported by Suzuki et al (13). However, the exact relationship between pancreatic stones and pancreatic carcinoma was unclear in these cases, unlike previously reported cases (12).

\section{Conclusion}

In conclusion, early diagnosis of pancreatic carcinoma is often difficult even in patients with chronic pancreatitis who have been followed for long periods. The survival time was short in pancreatic carcinoma patients with chronic pancreatitis. The following points should indicate that more careful imaging examinations are necessary: continuous severe pain different from previous attacks of pancreatitis, weight loss and appetite loss, large pancreatic stone, and changes in laboratory data, especially serum elastase 1 values, exacerbation of glucose intolerance, and tumor markers such as serum CA19-9. For the differential imaging diagnosis, the following procedures are important: careful follow-up observation of mass lesions, appearance of lymph node swelling, precise visualization of pancreatic ducts and blood vessels, and combined examination with biopsy.
This study was supported in part by a Grant-in-Aid for Cancer Research from the Public Health Bureau, Ministry of Health and Welfare, Japan. The paper was presented at the 8th Meeting of the International Association of Pancreatology in July 1998 Tokyo, Japan. The authers thank Miss K. Yoshitake for her secretarial assistance.

\section{References}

1) Lowenfels $\mathrm{AB}$, Maisonneuve $\mathrm{P}$, Cavallini $\mathrm{G}$, et al, and International Pancreatitis Study Group. Pancreatitis and the risk of pancreatic cancer. N Engl J Med 328: 1433-1437, 1993.

2) Yamaguchi K. Pancreatic carcinoma associated with chronic calcifying pancreatitis. Int J Pancreatol 12: 297-303, 1992.

3) Gambill EE. Pancreatitis associated with pancreatic carcinoma: a study of 26 cases. Mayo Clin Proc 46: 174-177, 1971.

4) Report of the Japan Pancreas Society Investigating Committee on chronic pancreatitis: Clinical diagnostic criteria for chronic pancreatitis. Jpn J Gastroenterol 80. 1863-1866, 1983 (in Japanese).

5) Homma T, Harada H, Koizumi M. Diagnostic criteria for chronic pancreatitis by the Japan Pancreas Society. Pancreas 15: 14-15, 1997.

6) Wakasugi $\mathrm{H}$, Ishikawa $\mathrm{H}$, Yasuda $\mathrm{D}$, et al. A case of pancreatic cancer with ascites-another diagnostic enigma IRYO (Jpn J National Med services) 46: 225-230, 1992 (Abstract in English).

7) Ikeda S. Clinical Pathology of Pancreatic Disease Viewed from the Pancreatic Duct Imaging. Igaku Shoin, Tokyo, 1991 (In Japanese).

8) Karlson B-M, Ekbom A, Josefsson S, McLaughlin JK, Fraumeni JF Jr, Nyrén $O$. The risk of pancreatic cancer following pancreatitis: an association due to confounding? Gastroenterology 113: 587-592, 1997.

9) Furuya $\mathrm{N}$, Kawa $\mathrm{S}$, Akamatsu T, Furihata K. Long-term follow-up of patients with chronic pancreatitis and $\mathrm{K}$-ras gene mutation detected in pancreatic juice. Gastroenterology 113: 593-598, 1997.

10) Suehara N, Mizumoto $K$, Tanaka $M$, et al. Telomerase activity in pancreatic juice differentiates ductal carcinoma from adenoma and pancreatitis. Clin Cancer Res 3: 2479-2483, 1997.

11) Bramhall SR. The use of molecular technology in the differentiation of pancreatic cancer and chronic pancreatitis. Int J Pancreatol 23: 83-100, 1998.

12) Machiki Y, Nimura Y, Kamiya J, et al. Clinicopathologic study on pancreatic cancer associated with pancreatic stones. Int J Pancreatol 22: $187-$ $191,1997$.

13) Suzuki T, Hayakawa T, Noda A, Aoki I, Inoue J. Carcinoma of the pancreas in association with pancreatolithiasis. Jpn J Gastroenterol 72: 15631568, 1975 (Abstract in English). 